\title{
Attenuation of the Electric Potential and Field in Disordered Systems
}

\author{
A. Singer, ${ }^{1}$ Z. Schuss, ${ }^{1}$ and R. S. Eisenberg ${ }^{2}$
}

Received October 12, 2004; accepted January 19, 2005

\begin{abstract}
We study the electric potential and field produced by disordered distributions of charge to see why clumps of charge do not produce large potentials or fields. The question is answered by evaluating the probability distribution of the electric potential and field in a totally disordered system that is overall electroneutral. An infinite system of point charges is called totally disordered if the locations of the points and the values of the charges are random. It is called electroneutral if the mean charge is zero. In one dimension, we show that the electric field is always small, of the order of the field of a single charge, and the spatial variations in potential are what can be produced by a single charge. In two and three dimensions, the electric field in similarly disordered electroneutral systems is usually small, with small variations. Interestingly, in two and three dimensional systems, the electric potential is usually very large, even though the electric field is not: large amounts of energy are needed to put together a typical disordered configuration of charges in two and three dimensions, but not in one dimension. If the system is locally electroneutralas well as globally electroneutral-the potential is usually small in all dimensions. The properties considered here arise from the superposition of electric fields of quasi-static distributions of charge, as in non-metallic solids or ionic solutions. These properties are found in distributions of charge far from equilibrium.
\end{abstract}

KEY WORDS: Screening; shielding; disordered charge.

\section{INTRODUCTION}

There is no danger of electric shock when handling a powder of salt or when dipping a finger in a salt solution, although these systems have huge

\footnotetext{
${ }^{1}$ Department of Applied Mathematics, Tel-Aviv University, Ramat-Aviv, 69978 Tel-Aviv, Israel; e-mail: amits@post.tau.ac.il and schuss@post.tau.ac.il

${ }^{2}$ Department of Molecular Biophysics and Physiology, Rush Medical Center, 1750 Harrison Street, Chicago, IL 60612, USA; e-mail: beisenbe@rush.edu
} 
numbers of positive and negative charges. It seems intuitively obvious that the alternating arrangement of charge in crystalline $\mathrm{Na}^{+} \mathrm{Cl}^{-}$should produce electric fields that add almost to zero; it also seems obvious that $\mathrm{Na}^{+}$and $\mathrm{Cl}^{-}$ions will move in solution to minimize their equilibrium free energy and produce small electrical potentials. But what about random arrangements of charge that occur in a random quasi-static arrangement of charge such as a snapshot of the location of ions in a solution? Tiny imbalances in charge distribution produce large potentials, so why does not a random distribution of charge produce large potentials, particularly if the distribution is not at thermodynamic equilibrium? Indeed, some arrangements of charge produce arbitrarily large potentials, but as we shall see, these distributions occur rarely enough that the mean and variance of stochastic distributions are usually finite and small. More specifically, we determine the conditions under which stochastic distributions of fixed charge produce small fields.

The quasi-static arrangements of charge can represent the fixed charge in amorphous non-metallic solids or snapshots of charge arrangement of ions in solution, due to their random (Brownian) motion. Our analysis does not apply to quantum systems, ${ }^{(1)}$ and in particular it fails if electrons move in delocalized orbitals, as in metals. Note that the random arrangements of charge considered here do not necessarily minimize free energy.

We consider the field and potential in overall electroneutral random configurations of infinitely many point charges. An infinite system of point charges is called totally disordered if the locations of the points and the charges are random, and it is called overall electroneutral if the mean charge is zero. The configurations of charge may be static or quasi-static, that is, time dependent, but varying sufficiently slowly to avoid electromagnetic phenomena: the electric potential is described by Coulomb's law alone. In one dimensional systems of this type, the potential is usually finite - even though the system usually contains an infinite number of positive and negative charges. Even if the system is disordered and spatially random, charges of the same sign do not clump together often enough to produce large fields or potentials, in one dimensional systems.

Our approach is stochastic. We ask how disordered can a random electroneutral system be, yet still have a small field or potential. We find the answer by evaluating the probability distribution of the electric potential and field of a disordered system of charges. We find that the electric field in a totally disordered one dimensional system is small whether the system is locally electroneutral or not. The potential behaves differently; it can be arbitrarily large in a one dimensional system, but it is usually small in electroneutral systems. 
In two or three dimensional disordered systems, the electric field is not necessarily small. We show that in such systems that are also electroneutral the field is usually small. The potential, however, is usually large, even if the system is electroneutral. Both potential and field are small, if the system is locally - as well as globally-electroneutral (see definition below) in one, two and three dimensions.

We consider several types of random arrays of charges: (a) A lattice with random distances between two nearest charges; (b) A lattice (of random or periodic structure) with a random distribution of positive and negative charges (charge \pm 1 ). Charges in the lattice need not alternate between positive and negative, nor need they be periodically distributed; (c) A lattice (of random or periodic structure) with random charge strengths. Not all charges are \pm 1 , but they are chosen from a set $q_{1}, q_{2}, \ldots, q_{n}$ with probabilities $p_{1}, p_{2}, \ldots, p_{n}$, respectively, such that

$$
\sum_{i=1}^{n} q_{i} p_{i}=0
$$

Equation (1) is our definition of electroneutrality in an infinite system.

We use renewal theory, ${ }^{(2)}$ perturbation theory, ${ }^{(3)}$ and saddle point approximation $^{(4)}$ to calculate the electric potential of one dimensional systems of charges and show that it is usually small. That is to say, the probability is small that the potential takes on large values. Thus, randomly distributed particles produce small potentials even in disordered systems in one dimension, if the system is electroneutral. The analysis of one dimensional systems requires the calculation of the probability density function (pdf) of weighted independent identically distributed (i.i.d.) sums of random variables. This pdf looks like the normal distribution near its center, but the tail distribution has the double exponential decay of the log-Weibull distribution. ${ }^{(5)}$ We conclude that the electric potential of totally disordered electroneutral one dimensional systems is necessarily small, comparable to that of a single charge.

Later in the paper, we define local electroneutrality precisely and show that two and three dimensional systems with local electroneutrality usually have small potentials, because the potential of a locally neutral system of charges decays like the potential of a point dipole, as $1 / r^{2}$. We show that the potential of typical totally disordered arrays of charges in two and three dimensions is infinite even if the system is electroneutral.

Historically, little attention seems to have been paid to quasi-static random arrangements of charge, although much attention has been paid to the equilibrium arrangements of mobile charge. In systems of mobile 
charges, such as liquids and ionic solutions, the decay of the electric potential may even be exponential, after the mobile charges assume their equilibrium distribution. The early theory of Debye-Hückel ${ }^{(6)}$ shows a nearly exponential decay (with distance from a given particle) of the average electric potential at equilibrium, originally found by solving the linearized Poisson-Boltzmann equation. In classical physics, perfect screening of multipoles (of all orders) occurs in both homogeneous and inhomogeneous systems at equilibrium in the thermodynamic limit, when boundary conditions at infinity are chosen to have no $\operatorname{effect}^{(7)}$ and there is no flux of any species. This type of screening in electrolytic solutions is produced by the equilibrium configuration of the mobile charges, ${ }^{(8,9)}$ which typically takes $100 \mathrm{ps}$ to establish (compared to the $10^{-16}$ time scale of most atomic motions). ${ }^{(10)}$ Many other systems are screened by mobile charges after they assume their equilibrium configuration of lowest free energy, ${ }^{(1)}$ such as ionic solutions, metals and semiconductors.

The spatial decay of potential in ionic solutions determines many of the properties of ionic solutions and is a striking example of screening or shielding. "Sum rules" of statistical mechanics ${ }^{(8,9)}$ describe these properties. These rules depend on the system assuming an equilibrium distribution, which can only happen if the charges are mobile.

We consider finite and infinite systems of charges which may or may not be mobile and which are not necessarily at equilibrium. We show that the potential of a finite disordered locally electroneutral system is attenuated to the potential of a single typical charge, whether the potential is evaluated inside or outside a finite system or in an infinite system. We note that the behavior of the electric potential and field outside the line or plane of the lattice can be analyzed in a straightforward manner by the methods developed below.

\section{A ONE-DIMENSIONAL IONIC LATTICE}

Consider a semi-infinite array of alternating electric charges $\pm q$ with a distance $d$ between neighboring charges. The electric potential $\Phi$ at a point $P$, located at a distance $R$ from and to the left of the first charge (see Fig. 1) is given by

$$
\begin{aligned}
\Phi & =\frac{q}{4 \pi \varepsilon_{0}}\left(\frac{1}{R}-\frac{1}{R+d}+\frac{1}{R+2 d}-\frac{1}{R+3 d}+\cdots\right) \\
& =\frac{q}{4 \pi \varepsilon_{0} R}\left(1-\frac{1}{1+a}+\frac{1}{1+2 a}-\frac{1}{1+3 a}+\cdots\right) \\
& =\frac{q}{4 \pi \varepsilon_{0} R} \sum_{n=0}^{\infty} \frac{(-1)^{n}}{1+n a},
\end{aligned}
$$




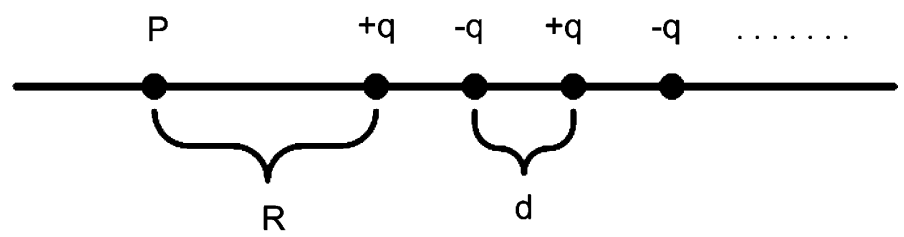

Fig. 1. A semi infinite lattice of alternating charges with a distance $d$ between neighboring charges. The point $P$ is located at a distance $R$ from and to the left of the first charge.

where $a=d / R$ is a dimensionless parameter. The series (2) is conditionally convergent, so it can be summed to any value by changing the order of summation. ${ }^{(13)}$ The order of summation reflects the order of construction of the system; different orders may lead to different potential energies of the system. However, the infinite series that determines the electric field

$$
E=\frac{q}{4 \pi \varepsilon_{0} R^{2}} \sum_{n=0}^{\infty} \frac{(-1)^{n}}{(1+n a)^{2}}
$$

is absolutely convergent, so the field does not depend on the order of summation of its defining series. Thus, all potentials differ from each other by a constant, which presumably reflects the different ways the charge distribution could be constructed, while having the same electric field. From here on, we consider the ordering in Eq. (2).

Setting $R=d(a=1)$ we find the potential at a vacant lattice point (to avoid infinite potentials) due to charges located at both directions of the infinite lattice is

$$
2 \Phi(R=d)=2 \frac{q}{4 \pi \varepsilon_{0} d} \sum_{n=1}^{\infty} \frac{(-1)^{n-1}}{n}=\frac{q}{4 \pi \varepsilon_{0} d} \cdot 2 \log 2 .
$$

The constant $2 \log 2$ is known as the Madelung constant of a one-dimensional lattice. $^{(12)}$

Next we find the asymptotic behavior of the potential $\Phi$ away from the semi-infinite lattice, that is for $R \gg d$, or equivalently $a \ll 1$. The following analysis is independent of the order of summation of the series (2). Clearly, the infinite sum in Eq. (2) converges, because it is an alternating sum with a decaying general term. We expand the potential for $a \ll 1$ (away from the lattice) in the asymptotic form

$$
\Phi=\frac{q}{4 \pi \varepsilon_{0}} \frac{1}{R}\left(V_{0}+a V_{1}+a^{2} V_{2}+\cdots\right) .
$$


The effect of the first charge can be separated from all the others,

$$
\Phi=\frac{q}{4 \pi \varepsilon_{0}} \frac{1}{R}-\frac{q}{4 \pi \varepsilon_{0}} \frac{1}{R+d}\left(V_{0}+\tilde{a} V_{1}+\tilde{a}^{2} V_{2}+\cdots\right),
$$

where

$$
\tilde{a}=\frac{d}{R+d}=\frac{a}{1+a} .
$$

Comparing Eqs. (3) and (4) we obtain

$$
V_{0}+a V_{1}+a^{2} V_{2}+\cdots=1-\frac{1}{1+a}\left[V_{0}+\frac{a}{1+a} V_{1}+\left(\frac{a}{1+a}\right)^{2} V_{2}+\cdots\right]
$$

The coefficients $V_{0}, V_{1}, \ldots$ are found by equating the coefficients of like powers of $a$. In particular, we find that $V_{0}=1 / 2, V_{1}=1 / 4, V_{2}=0$, so the potential has the asymptotic form

$$
\Phi=\frac{q}{4 \pi \varepsilon_{0}} \frac{1}{R}\left[\frac{1}{2}+\frac{1}{4} a+O\left(a^{3}\right)\right] .
$$

All coefficients $V_{n}$ can easily be computed in a similar fashion. This result also determines the rate at which the potential far away reaches its limiting value, $\frac{1}{2} \frac{q}{4 \pi \varepsilon_{0} R}$. The divergent series for $x=1$ has the value $V_{0}=\frac{1}{2}$ if interpreted as a limit using the Abel sum ${ }^{(13)}$

$$
1-1+1-1+1-1+\cdots=\lim _{x \rightarrow 1^{-}} \sum_{n=0}^{\infty}(-1)^{n} x^{n}=\lim _{x \rightarrow 1^{-}} \frac{1}{1+x}=\frac{1}{2} .
$$

We note that the asymptotic expansion (5) can also be found directly from the differential equation that the sum

$$
y(x)=\sum_{n=0}^{\infty} \frac{(-1)^{n}}{1+n a} x^{n}
$$

satisfies $^{(14)}$

$$
a x y^{\prime}+y=\frac{1}{1+x},
$$


with initial condition $y(0)=1$. The asymptotic form of $y(x)$ can easily be found by standard methods. ${ }^{(3)}$ In particular,

$$
\lim _{x \rightarrow 1-} y(x)=\sum_{n=0}^{\infty} \frac{(-1)^{n}}{1+n a} .
$$

The physical interpretation of the asymptotic expansion (5) is that the electric potential away from an infinite lattice of charged particles is about the same as if half a single charge were located at the origin. The spatial arrangement of the lattice attenuates the effect of its charge. The potential near the lattice is determined by a few of the nearest charges and the contribution of the remaining charges reduces to that of a half charge placed at a distance $R \gg d$. Obviously, as $R \rightarrow 0$ the potential becomes infinite, approaching the potential produced by just the nearest charge.

\section{ONE-DIMENSIONAL RANDOM IONIC LATTICE}

We turn now to solids in which the charges are distributed randomly in several different ways. First, consider a semi-infinite lattice of electric charges, in which the sign of each charge is determined randomly by a flip of a fair coin. That is, the charges that are located at the lattice points $X_{n}(n=0,1,2, \ldots)$ are independent Bernoulli random variables that take the values \pm 1 with probability $1 / 2$. The electric potential of this random lattice is given by

$$
\Phi=\frac{q}{4 \pi \varepsilon_{0} R} \sum_{n=0}^{\infty} \frac{X_{n}}{1+n a} .
$$

Some discussion of the nature of convergence of the series (7) is needed at this point. The convergence of the sum of variances means that the partial sums converge in $L^{2}$ with respect to the probability measure, so the sum (7) exists as a random variable $\Phi \in L^{2}$, whose variance is the sum of the variances. Now, the Cauchy-Schwarz inequality implies that $\Phi \in L^{1}$, so $\langle\Phi\rangle=0$. Note that (7) also converges with probability $1 .{ }^{(15)}$

We use fair coin tossing to maintain the condition of global electroneutrality, though arbitrary long runs of positive or negative charges occur in this distribution. Thus some realizations of the sequence $X_{n}$ have runs ('clumps') of substantial net charge and potential. The standard deviation of the net charge in a region gives some feel for the size of the clumps. The standard deviation in the net charge of a region containing $N$ charges is $q \sqrt{N}$. For large values of $N$, substantial regions are not charge neutral. 
The condition of local charge neutrality (defined later) is violated for many of the realizations of charge in this distribution.

Note that a particular set of $X_{n}$ can produce an infinite potential, despite our general conclusions. If, for example, $X_{n}=1$ for all $n$, the electric potential becomes infinite (see Eq. (7)), because $\sum_{n=0}^{\infty} \frac{1}{1+n a}=\infty$. Nonetheless, the $L^{2}$ convergence of (7) implies that the probability that (7) is infinite is 0 . In other words, even though the potential is infinite for a particular set of $X_{n}$, the potential is finite with probability 1 . This is a striking example of the attenuation of the electric field, even without mobile charge. The attenuation of the potential produced by some 'clumpy' configurations of charges occurs even though there is no correlation in position, and there is no motion whatsoever.

The electric field, given by

$$
E=-\frac{q}{4 \pi \varepsilon_{0} R^{2}} \sum_{n=0}^{\infty} \frac{X_{n}}{(1+n a)^{2}},
$$

remains finite for all realizations of $X_{n}$, because the sum

$$
S=\frac{q}{4 \pi \varepsilon_{0} R^{2}} \sum_{n=0}^{\infty} \frac{1}{(1+n a)^{2}}
$$

converges.

The electric field is bounded (above and below) by $S$ and so there is zero probability that the function is outside the interval $(-S, S)$. The pdf of the electric field is compactly supported, even when all charges are positive (or negative). The electric field - unlike the potential - is attenuated even if the net charge of the system is not zero, taken as a whole. The standard deviation of the field is $\frac{q}{4 \pi \varepsilon_{0} R^{2}}\left\{\sum_{n=0}^{\infty} \frac{1}{(1+n a)^{4}}\right\}^{1 / 2}$, which is of the order of the field of a single charge at a distance $R$.

\subsection{Moments}

The expected value of $\Phi$ is $\langle\Phi\rangle=0$, as mentioned above. The variance of $\Phi$ is given by

$$
\operatorname{Var}(\Phi)=\left(\frac{q}{4 \pi \varepsilon_{0} R}\right)^{2} \sum_{n=0}^{\infty} \frac{1}{(1+n a)^{2}}
$$


A vacant lattice point in an infinite (not semi-infinite) lattice corresponds to $R=d$ for both the charges to the right and to the left. It follows that the variance of the potential there is twice that given in (8) with $a=1$, that is,

$$
\operatorname{Var}(\Phi)=2\left(\frac{q}{4 \pi \varepsilon_{0} d}\right)^{2} \sum_{n=1}^{\infty} \frac{1}{n^{2}}=2\left(\frac{q}{4 \pi \varepsilon_{0} d}\right)^{2} \frac{\pi^{2}}{6},
$$

so that the standard deviation is

$$
\sigma_{\Phi}=\frac{q}{4 \pi \varepsilon_{0} d} \frac{\pi}{\sqrt{3}} .
$$

As expected, the constant $\pi / \sqrt{3}$ is larger than the Madelung constant $2 \log 2$ of the periodic lattice, because the potential of the disordered system is larger than that of the ordered one.

Away from the semi infinite lattice, i.e., for $a \ll 1$, we can approximate the variance (8) by the Euler-Maclaurin formula, which replaces the sum by an integral,

$$
\begin{aligned}
\operatorname{Var}(\Phi) & =\left(\frac{q}{4 \pi \varepsilon_{0} R}\right)^{2}\left(\int_{0}^{\infty} \frac{1}{(1+a x)^{2}} d x+\frac{1}{2}+O(a)\right) \\
& =\left(\frac{q}{4 \pi \varepsilon_{0} R}\right)^{2}\left(\frac{1}{a}+\frac{1}{2}+O(a)\right),
\end{aligned}
$$

so the standard deviation is

$$
\left.\sigma_{\phi}\right|_{R}=\frac{q}{4 \pi \varepsilon_{0} \sqrt{d R}}(1+O(a))
$$

The decay law of $1 / \sqrt{R}$ is more gradual than the decay law $1 / R$ of a single charge.

\subsection{The Electrical Potential as a Weighted i.i.d. Sum}

The potential (7) is a weighted sum of the form $\sum a_{n} X_{n}$, where $X_{n}$ are i.i.d. random variables. The distribution of potential is generally not normal. For example, consider the weighted sum $\sum_{n=1}^{\infty} 2^{-n} X_{n}$, where $X_{n}$ are the same Bernoulli random variables. This weighted sum represents the uniform distribution in the interval $[-1,1]$. It is, in fact equivalent to the binary representation of real numbers in the interval. Not only does this 
distribution not look like the Gaussian distribution for small deviations, it does not look at all Gaussian for large deviations. In fact, this distribution has compact support. It is zero outside a finite interval, without the tails of the better endowed Gaussian. Other unusual limit distributions can be easily obtained from sums of the form (7). For example, the weighted sum $\sum_{n=1}^{\infty} 3^{-n} X_{n}$ is equivalent to the uniform distribution on the Cantor "middle thirds" set ${ }^{(16)}$ in $[-1,1]$, whose Lebesgue measure (length) is 0 .

Note that the sum

$$
\sum_{n=0}^{\infty} \frac{X_{n}}{(1+n a)^{1+\varepsilon}}
$$

has compact support for every $\varepsilon>0$, because the series

$$
\sum_{n=0}^{\infty} \frac{1}{(1+n a)^{1+\varepsilon}}
$$

converges for every $\varepsilon>0$. In our case $\varepsilon=0$, so that the limit distribution does not necessarily have compact support. Nonetheless, we expect that the probability distribution function of the potential will have tails that decay steeply, even steeper than those of the normal distribution.

\subsection{Large and Small Potentials. The Saddle Point Approximation}

The existence of the first moment of the sum (7) depends on its tail distribution, which we calculate below by the saddle point method. ${ }^{(4)}$ That is, we calculate the chance of finding a pinch of (non-crystalline) salt with a very large potential. For a potential $\Phi$ defined in Eq. (7), we denote the pdf of $\left(\frac{q}{4 \pi \varepsilon_{0} R}\right)^{-1} \Phi$ by $f(x)$. The Fourier transform $\hat{f}(k)$ of this pdf is given by the infinite product

$$
\hat{f}(k)=\prod_{n=0}^{\infty} \cos \left(\frac{k}{1+n a}\right),
$$

which is an entire function in the complex plane, because the general term is $1+O\left(n^{-2}\right)$. The inverse Fourier transform recovers the pdf

$$
f(x)=\frac{1}{2 \pi} \int_{-\infty}^{\infty} \hat{f}(k) e^{i k x} d k
$$


which we want to evaluate asymptotically for large $x$. Setting

$$
g(k, x)=\sum_{n=0}^{\infty} \log \cos \left(\frac{k}{1+n a}\right)+i k x,
$$

we write

$$
f(x)=\frac{1}{2 \pi} \int_{-\infty}^{\infty} \exp \{g(k, x)\} d k .
$$

The saddle point is the point $k$ for which $\frac{d}{d k} g(k, x)=0$. Differentiating Eq. (15) with respect to $k$, we find that

$$
\frac{d}{d k} g(k, x)=-\sum_{n=0}^{\infty} \frac{\tan \left(\frac{k}{1+n a}\right)}{1+n a}+i x .
$$

We look for a root of the derivative on the imaginary axis, and substitute $k=i s$. The vanishing derivative condition of the saddle point method is then

$$
x=\sum_{n=0}^{\infty} \frac{\tanh \left(\frac{s}{1+n a}\right)}{1+n a} .
$$

The infinite sum on the right hand side represents a monotone increasing function of $s$ in the interval $0<s<\infty$, so Eq. (18) has exactly one solution for every $x$. Near the saddle point $k=i s$, we approximate $g(k)$ by its Taylor expansion up to the order

$$
g(k) \approx g(i s)+\frac{1}{2} \frac{d^{2}}{d k^{2}} g(i s)(k-i s)^{2},
$$

to find the leading order term of the full asymptotic expansion (derivatives of higher order of the Taylor expansion can be used to find all terms 


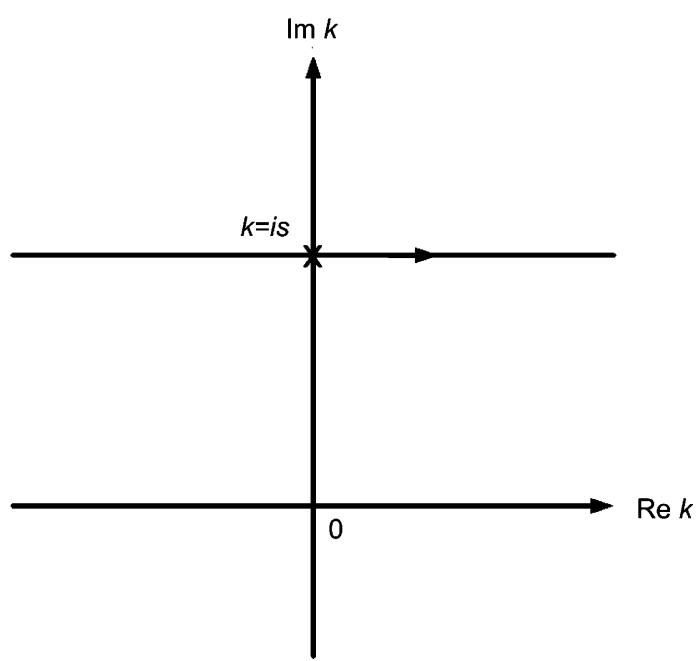

Fig. 2. The integration contour passes through the saddle point $k=i s$ in the complex plane.

of the asymptotic expansion. $\left.{ }^{(17)}\right)$ We use the Cauchy integral formula to calculate our Fourier integral (16) on the line parallel to the real $k$ axis through $k=i s$ (see Fig. 2)

$$
\begin{aligned}
f(x) & \approx \frac{1}{2 \pi} e^{g(i s)} \int_{-\infty}^{\infty} \exp \left\{\frac{1}{2} g^{\prime \prime}(i s)(k-i s)^{2}\right\} d k \\
& =\frac{1}{2 \pi} e^{g(i s)} \int_{-\infty}^{\infty} \exp \left\{g^{\prime \prime}(i s) \frac{z^{2}}{2}\right\} d z=\frac{e^{g(i s)}}{\sqrt{-2 \pi g^{\prime \prime}(i s)}} .
\end{aligned}
$$

Equation (18) has no analytic solution, so we construct asymptotic approximations for large and small values of $s$ separately.

\subsection{Tail Asymptotics}

Throughout this subsection we assume that $a$ is small and $s$ is large and we find the tail asymptotics of the pdf away from the system (for $a \ll 1$ ). For $s \gg 1$ the Euler-Maclaurin sum formula gives 


$$
x=\int_{0}^{\infty} \frac{\tanh \left(\frac{s}{1+a x}\right)}{1+a x} d x+\frac{1}{2} \tanh s+O(a)
$$

Substituting $z=\frac{s}{1+a x}$, we obtain

$$
x=\frac{1}{a} \int_{0}^{s} \frac{\tanh z}{z} d z+\frac{1}{2} \tanh (s)+O(a) .
$$

Writing

$$
\begin{aligned}
\int_{0}^{s} \frac{\tanh z}{z} d z & =\int_{0}^{1} \frac{\tanh z}{z} d z+\int_{1}^{s} \frac{\tanh z-1}{z} d z+\int_{1}^{s} \frac{d z}{z} \\
& =\log s+\int_{0}^{1} \frac{\tanh z}{z} d z+\int_{1}^{\infty} \frac{\tanh z-1}{z} d z+O\left(e^{-2 s}\right),
\end{aligned}
$$

we obtain (22) in the form

$$
a x=\log s+C+\frac{a}{2}+O\left(a^{2}, e^{-2 s}\right)
$$

where the constant $C$ is given by

$$
C=\int_{0}^{1} \frac{\tanh z}{z} d z+\int_{1}^{\infty} \frac{\tanh z-1}{z} d z
$$

Exponentiation of Eq. (23) gives the location of the saddle point asymptotically for small $a$ and large $s$ as

$$
s=e^{a x-C-a / 2+O\left(a^{2}, e^{-2 s}\right)} .
$$

The saddle point approximation (20) requires the evaluation of $g$ and its second derivative at $k=i s$. The Euler-Maclaurin sum formula gives

$$
\begin{aligned}
g(i s) & =\sum_{n=0}^{\infty} \log \cosh \left(\frac{s}{1+n a}\right)-s x \\
& =\frac{s}{a} \int_{0}^{s} \frac{\log \cosh z}{z^{2}} d z+\frac{1}{2} \log \cosh s-s x+O(a s)
\end{aligned}
$$




$$
\begin{aligned}
= & \frac{s}{a}\left(\int_{0}^{1} \frac{\log \cosh z}{z^{2}} d z+\int_{1}^{s} \frac{d z}{z}+\int_{1}^{\infty} \frac{\log \cosh z-z}{z^{2}} d z+O\left(\frac{1}{s}\right)\right) \\
& +\frac{s}{2}-\frac{\log 2}{2}-s x+O(\text { as }) .
\end{aligned}
$$

Using Eqs (23) and (24), we find

$$
g(i s)=C_{1} \frac{s}{a}-\frac{\log 2}{2}+O\left(a, \frac{1}{a}, a s\right),
$$

where

$$
\begin{aligned}
C_{1}= & \int_{0}^{1} \frac{\log \cosh z}{z^{2}} d z+\int_{1}^{\infty} \frac{\log \cosh z-z}{z^{2}} d z \\
& -\int_{0}^{1} \frac{\tanh z}{z} d z-\int_{1}^{\infty} \frac{\tanh z-1}{z} d z,
\end{aligned}
$$

and integration by parts shows that $C_{1}=-1$. It follows that

$$
g(i s)=-\frac{s}{a}-\frac{\log 2}{2}+O\left(a, \frac{1}{a}, a s\right) .
$$

The second derivative of $g$ is evaluated in a similar fashion

$$
\begin{aligned}
& \left.\frac{d^{2}}{d k^{2}} g(k)\right|_{k=i s}=-\sum_{n=0}^{\infty} \frac{1-\tanh ^{2}\left(\frac{s}{1+n a}\right)}{(1+n a)^{2}} \\
= & -\frac{1}{a s} \int_{0}^{s}\left(1-\tanh ^{2} z\right) d z-\frac{1}{2}\left(1-\tanh ^{2} s\right)+O\left(a s e^{-2 s}\right) \\
= & -\frac{\tan \mathrm{h} s}{a s}+O\left(a s e^{-2 s}, e^{-2 s}\right) \\
= & -\frac{1}{a s}+O\left(\text { as }, 1, \frac{1}{a s}\right) e^{-2 s} .
\end{aligned}
$$

Substitution of (28), (29), and (25) into the saddle point approximation (20) gives

$$
f(x) \approx \frac{\sqrt{a}}{2 \sqrt{\pi}} e^{\frac{1}{2}(a x-C-a / 2)} e^{-\frac{1}{a} e^{a x-C-a / 2},}
$$


where the constant $C=0.8187801402 \ldots$ is given by Eq. (24). Therefore, the small $a$ and large $s$ approximation to the tail of the pdf of $\Phi$ is given by

$$
\begin{aligned}
f_{\Phi}(x) \sim \frac{4 \pi \varepsilon_{0} R}{q} \frac{\sqrt{a}}{2 \sqrt{\pi}} \exp \{ & \frac{1}{2}\left(\frac{4 \pi \varepsilon_{0} d}{q} x-C-a / 2\right) \\
& \left.-\frac{1}{a} \exp \left\{\frac{4 \pi \varepsilon_{0} d}{q} x-C-a / 2\right\}\right\}, \quad x \rightarrow \infty .
\end{aligned}
$$

It follows from Eq. (31) that the pdf decays to zero as a double exponential as $x \rightarrow \infty$, which implies that all moments exist. This decay is similar to the extreme value or the log-Weibull (Gumbel) distributions. ${ }^{(5)}$ The compact support of the distributions of convergent series is replaced here with a steep decay. Note also that the decay becomes steeper further away from the system, as expected, because the pre-exponential factor of the inner exponent is $1 / a=R / d$.

For small $x$ the pdf can be approximated by a zero mean Gaussian with variance $\operatorname{Var}(\Phi)$, which for small $a$ is

$$
f_{\Phi}(x) \sim \frac{4 \pi \varepsilon_{0}}{q} \sqrt{\frac{R d}{2 \pi}} \exp \left\{-\frac{R d}{2}\left(\frac{4 \pi \varepsilon_{0} x}{q}\right)^{2}\right\}, \quad x \rightarrow 0
$$

Near its center, the distribution looks like a Gaussian with a standard deviation that decays like $1 / \sqrt{R}$, in agreement with Eq. (12). We conclude that the pdf looks normal near its center, but, far away from there, it decays to zero much more steeply, rather like a cutoff. This conclusion is the answer to the question posed in subsection 3.2 about the normality of weighted sums of i.i.d. random variables. The non-Gaussian tails of the distribution are characteristic of large deviations. ${ }^{(4)}$

\section{RANDOM DISTANCES}

Consider a one-dimensional system of alternating charges without the restriction of equal distance between successive charges. In particular, we assume a renewal model, in which the distances between two neighboring charges are non-negative i.i.d random variables with pdf $f(l)$ and finite expectation value

$$
d=\int_{0}^{\infty} l f(l) d l<\infty
$$


The potential of this random system is also a random variable.

We show below that away from the system the mean value of the potential $\bar{V}$ has the asymptotic form

$$
\bar{V}=\frac{q}{4 \pi \varepsilon_{0} R}\left(\frac{1}{2}+O(a)\right),
$$

where $a=d / R$. Equation (33) defines the attenuation produced by the configuration of charges. The mean potential of the system is produced by (in effect) half a charge. We note that the value $1 / 2$ is exactly the same for both random and non-random systems of alternating charges (Eq. (5)). We first note that

$$
\operatorname{Pr}\{V(R)=V\}=\int_{0}^{\infty} f(l) \operatorname{Pr}\left\{V(R+l)=V^{*}-V\right\} d l,
$$

where $V^{*}=\frac{q}{4 \pi \varepsilon_{0} R}$. To find the mean value, we multiply (34) by $V$ and integrate (note that $0 \leqslant V \leqslant V^{*}$ ), and then change the order of integration

$$
\begin{aligned}
\bar{V}(R) & =\int_{0}^{V^{*}} V d V \int_{0}^{\infty} f(l) \operatorname{Pr}\left\{V(R+l)=V^{*}-V\right\} d l \\
& =\int_{0}^{\infty} f(l) d l \int_{0}^{V^{*}} V \operatorname{Pr}\left\{V(R+l)=V^{*}-V\right\} d V \\
& =V^{*}-\int_{0}^{\infty} f(l) d l \int_{0}^{V^{*}} V \operatorname{Pr}\{V(R+l)=V\} d V \\
& =V^{*}-\int_{0}^{\infty} f(l) \bar{V}(R+l) d l .
\end{aligned}
$$

We look for an asymptotic expansion of the form

$$
\bar{V}(R)=\frac{q}{4 \pi \varepsilon_{0} R}\left(\bar{V}_{0}+a \bar{V}_{1}+a^{2} \bar{V}_{2}+\cdots\right) .
$$

Substituting this asymptotic expansion into (35) gives $\bar{V}_{0}=1 / 2$ for the $O$ (1) term, because

$$
1-a \leqslant \int_{0}^{\infty} f(l) \frac{R}{R+l} d l \leqslant 1 .
$$

The first inequality is due to the inequality $\frac{1}{1+x} \geqslant 1-x$. Hence (33) follows. 


\section{DIMENSIONS HIGHER THAN ONE}

\subsection{The Condition of Global Electroneutrality}

In dimensions higher than one, global electroneutrality is enough to dramatically attenuate the electric field, but it is not enough to produce a small potential, as shown below.

Consider the electric potential at a vacant site of random charges located at the points of a $2 \mathrm{D}$ square lattice

$$
\Phi=\sum_{(n, m) \neq(0,0)} \frac{X_{n m}}{\sqrt{n^{2}+m^{2}}} .
$$

The variance of $\Phi$ is

$$
\operatorname{Var}(\Phi)=\sum_{(n, m) \neq(0,0)} \frac{1}{n^{2}+m^{2}}=\infty
$$

The infinite value of the variance means that arbitrarily large potentials can occur with high probability. That is, the electric potential is not attenuated. The divergence of the variance of the potential of three-dimensional systems is even steeper. Therefore, attenuation of the potential of totally disordered systems can occur in two or three-dimensional systems only if some correlation is introduced into the distribution of the locations of the charges. If, for example, the signs of all charges alternate, as in a real $\mathrm{Na}^{+} \mathrm{Cl}^{-}$crystal, the distribution of potential will be dramatically different, and greatly attenuated, compared to a two or threedimensional system in which many charges of one sign are clumped together.

The condition of global electroneutrality is enough to ensure the dramatic attenuation of the electric field. Indeed, consider a three-dimensional cubic lattice of random charges. The $z$-component of the electric field at a vacant lattice point is

$$
E_{z}=\sum_{(n, m, l) \neq(0,0,0)} \frac{X_{n m l} \cos \left(\frac{n}{\sqrt{n^{2}+m^{2}+l^{2}}}\right)}{n^{2}+m^{2}+l^{2}} .
$$


The variance of $E_{z}$ is finite,

$$
\operatorname{Var}\left(E_{z}\right)=\sum_{(n, m, l) \neq(0,0,0)} \frac{\cos ^{2}\left(\frac{n}{\sqrt{n^{2}+m^{2}+l^{2}}}\right)}{\left(n^{2}+m^{2}+l^{2}\right)^{2}}<\infty,
$$

because convergence is determined by the integral

$$
2 \pi \int_{0}^{\pi} \cos ^{2} \theta \sin \theta d \theta \int_{d}^{\infty} \frac{1}{r^{4}} r^{2} d r<\infty .
$$

The large potential means that much work has to be done to create the given spatial configuration of the charges, however, the resulting field remains usually small.

\subsection{The Condition of Local Electroneutrality}

Here we show that the condition of local electroneutrality implies the attenuation of the potential in two and three dimensions. For example, the potential of a two or three-dimensional lattice of extended dipoles is finite with probability 1 , if the orientation of dipoles is distributed independently, identically, and uniformly on the unit sphere (see Fig. 3).

Paraphrasing (in ref. 18, p. 136), we say that a (net) charge distribution $\rho(\boldsymbol{x})$ has local charge neutrality if the (net) charge inside a sphere of radius $R$ falls with increasing $R$ faster than any power, that is, for any $\boldsymbol{x}$

$$
\lim _{R \rightarrow \infty} R^{n} \int_{|\boldsymbol{x}-\boldsymbol{y}|<R} \rho(\boldsymbol{y}) d \boldsymbol{y}=0 \text { for all } n>0 .
$$

On a lattice, the number of charges that are assigned to each lattice point can be larger than in our example of dipoles (Fig. 3), thus forming multipoles. The Debye-Hückel distribution also satisfies the local charge neutrality condition.

The potential of a single lattice point can then be written as an expansion in spherical harmonics, if the charges of each multipole are contained in a single lattice box. It can be also expanded, if the charge density of each multipole decays sufficiently fast, as ${ }^{(18)}$

$$
\Phi_{0,0,0}(\boldsymbol{x})=\frac{1}{4 \pi \varepsilon_{0}} \sum_{l=0}^{\infty} \sum_{m=-l}^{l} \frac{1}{2 l+1} q_{l m} \frac{Y_{l m}(\theta, \phi)}{r^{l+1}},
$$




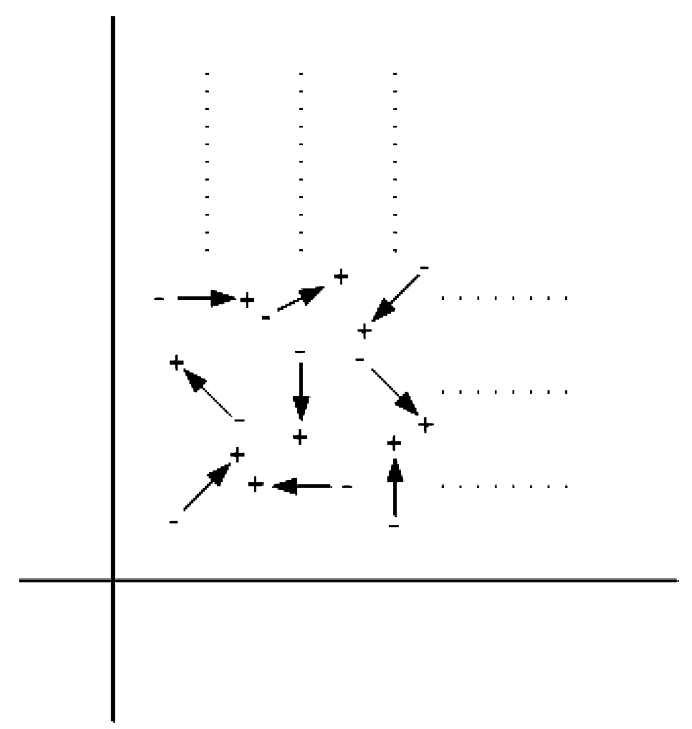

Fig. 3. Two-dimensional lattice of dipoles of randomly chosen orientations produce attenuation due to the condition of local electroneutrality.

where $q_{l m}$ are the multipole moments. In particular, the zeroth order multipole moment is

$$
q_{00}=\frac{1}{\sqrt{4 \pi}} \int \rho(\boldsymbol{y}) d \boldsymbol{y}=0,
$$

by the condition of local electroneutrality (41): the far potential due to a single lattice point decays as $1 / r^{2}$ (or steeper). The coefficients $q_{l m}$ assigned to each lattice point are randomized as in the previous sections so their mean value vanishes, meaning that there is no preferred orientation in space. (Compare the example of dipoles which do not have a preferred orientation.) The mean value of the potential of the entire lattice is then $\langle\Phi\rangle=0$. The variance is given by

$$
\operatorname{Var}(\Phi)=\sum_{i j k} \operatorname{Var}\left(\Phi_{i j k}\right),
$$

where $\Phi_{i j k}$ is the potential of the charge at lattice point $(i, j, k)$. The potential decays as $1 / r^{2}$ (or steeper); therefore the variance decays as $1 / r^{4}=1 /\left(i^{2}+j^{2}+k^{2}\right)^{2}$ (or steeper). The convergence of the infinite sum 
(44) is determined by the convergence of the integral

$$
\int_{r>d} \frac{1}{r^{4}} d V=4 \pi \int_{d}^{\infty} \frac{1}{r^{2}} d r=\frac{4 \pi}{d}<\infty .
$$

Thus, the variance of the potential is finite and we have shown that local electroneutrality produces a dramatic attenuation of potential. As above, the potential away from a charge is usually of the order of the potential of a single charge.

\subsection{The Liquid State}

Screening in the liquid state involves at least three phenomena. (1) The movement of charge to a distribution of minimal free energy. (2) The properties of a static charge distribution with minimal free energy. (3) The properties of any charge distribution.

If the charge correlation function $\rho(\boldsymbol{x})$ minimizes free energy, and is at equilibrium, as in ionic solutions, the far field potential is strongly screened. However, the relaxation into such a state takes time, typically picosecond to nanosecond in an ionic solution under biological conditions (see measurements reported in ref. 10, and theory summarized in ref. 19). As long as local charge neutrality exists during the relaxation period, the potential changes from attenuated (as described above) to exponentially screened, as equilibrium is reached. In fact, the spread of potential in ionic solutions has the curious property that it is much less shielded at short times than at long times; potentials on the (sub) femtosecond time scale of atomic dynamics spread macroscopic distances while potentials on long time scales spread only atomic distances. Specifically, potentials on a time scale greater than nano or microseconds spread a few Debye lengths, only a nanometer or so under biological conditions, although potentials on a femtosecond time scale can spread arbitrarily far depending on the configuration of dielectrics at boundaries that govern the violations of local electroneutrality. To make this verbal analysis of fast phenomena rigorous, the potentials and fields should be computed from Maxwell's equations, not Coulomb's law.

Non-equilibrium fluctuations may violate local charge neutrality, therefore field fluctuations can be large. For example, in systems which are not locally electroneutral, potential can spread a long way, as in the telegraph, ${ }^{(20)}$ Kelvin's transatlantic cable, or the axons of nerve cells. ${ }^{(21)}$ In such systems, d.c. potential spreads arbitrarily far-kilometers in telegraphs; thousands of kilometers in the transatlantic cable; centimeters in a squid nerve filled with salt water - even if an abundance of ions $\left(\approx 10^{23}\right)$ 
are present. Local electroneutrality is violated in such systems (at the insulating boundary which separates the inside and outside of the cable, e.g., the cell membrane) and that violation allows large far field potentials.

\section{SUMMARY AND DISCUSSION}

Global electroneutrality ensures the dramatic attenuation of the electric potential and field of a one-dimensional system of charges. Even if local electroneutrality is violated, and the local net charge is not zero, the potential remains finite in these one-dimensional systems, even in a random lattice that includes arbitrarily long strings of equal charges. We have shown that the distribution of the weighted sum of i.i.d. random variables that define the one-dimensional electric potential is almost normal near its center, but has very steep double exponentially decaying tails. The distances between neighboring charges can also be random, without changing the attenuation effect. In higher dimensions, global electroneutrality is sufficient to dramatically attenuate the electric field, but not the potential. However, local electroneutrality ensures a small potential in two and three dimensions, so the electric potential and field is short range in one, two, and three dimensions, if the systems are locally electroneutral.

\section{ACKNOWLEDGMENT}

The comments of David Ferry, Mark Ratner and Stuart Rice were most helpful. This research was partially supported by research grants from the Israel Science Foundation, US-Israel Binational Science Foundation, and the NIH Grant No. UPSHS 5 RO1 GM 067241.

\section{REFERENCES}

1. D. C. Brydges and Ph. A. Martin, Coulomb systems at low density: a review, J. Stat. Phys. 96(5/6):1163-1330 (1999).

2. S. Karlin and H. M. Taylor, A Second Course in Stochastic Processes (Academic Press, New York, 1981).

3. C. M. Bender and S. A. Orszag, Advanced Mathematical Methods for Scientists and Engineers (Springer, New York, 1999).

4. J. L. Jensen, Saddlepoint Approximations (Oxford Statistical Science Series, 16) (Oxford University Press, 1995).

5. V. Rothschild and N. Logothetis Probability Distributions (John Wiley, New York, 1985).

6. J. M. G. Barthel and H. Baumgartel (ed), H. Krienke, Physical Chemistry of Electrolyte Solutions: Modern Aspects (Steinkopf, Dietrich Pub. 1998).

7. D. A. McQuarrie, Statistical Mechanics (Harper and Row, NY, 1976).

8. D. Henderson (ed), Fundamentals of Inhomogeneous Fluids (Marcel Dekker, New York, 1992). 
9. P. A. Martin, Sum Rules in Charged Fluids, Rev. Mod. Phys. 60:1076-1127 (1988).

10. J. Barthel, R. Buchner, and M. Münsterer, Electrolyte Data Collection Vol. 12, Part 2: Dielectric Properties of Water and Aqueous Electrolyte Solutions. Frankfurt am Main, DECHEMA, 1995.

11. J. N. Chazalviel, Coulomb Screening by Mobile Charges (Birkhäuser, Boston, 1999).

12. C. Kittel, Introducation to Solid State Physics, 7th Ed. (John Wiley and Sons, New York, 1996).

13. K. Knopp, Theory and Application of Infinite Series (Dover, NY, 1990).

14. P. Mohazzabi and T. A. Fournelle, Evaluation of Ill-Behaved Power Series, Am. Math. Monthly 111(4):308-321 (April 2004).

15. L. Breiman, Probability (Classics in Applied Mathematics, No.7) (SIAM Publications (Reprint edition) 1992).

16. E. W. Weisstein et al. "Cantor Set." From MathWorld-A Wolfram Web Resource. http://mathworld.wolfram.com/CantorSet.html

17. G. F. Carrier, M. Krook and C. E. Pearson, Functions of a Complex Variable (McGrawHill, NY, 1966).

18. J. D. Jackson, Classical Electrodymnics, 2nd Ed. (Wiley, NY, 1975).

19. D. Knödler, W. Dieterich, C. Lonsky and A. Nitzan, Nonlinear relaxation and solvation dynamics in a Coulomb lattice gas, J. Chem. Phys. 102(1):465-470 (1995).

20. M. S. Ghausi and J. J. Kelly Introduction to Distributed-Parameter Networks (New York, Holt Rinehart \& Winston, 331, 1968).

21. J. J. B. Jack, D. Noble, and R. W. Tsien, Electric Current Flow in Excitable Cells (New York, Oxford, Clarendon Press, 1975). 